\title{
Klein-Gordon Equation and Wave Function for Free Particle in Rindler Space-Time
}

\author{
Sangwha-Yi* \\ Department of Math, Taejon University 300-716, South Korea
}

*Corresponding Author: Sangwha-Yi, Department of Math, Taejon University 300-716, South Korea

Abstract: Klein-Gordon equation is a relativistic wave equation. It treats spinless particle. The wave function cannot use as a probability amplitude. We made Klein-Gordon equation in Rindler space-time. In this paper, we make free particle's wave function as the solution of Klein-Gordon equation in Rindler space-time.

Keywords: Wave Function; Free Particle; Klein-Gordon equation Rindler Space-time;

PACS Number: 03.30. $+p, 03.65$

\section{INTRODUCTION}

At first, Klein-Gordon equation is for free particle field $\phi$ in inertial frame.

$\frac{m^{2} c^{2}}{\hbar^{2}} \phi+\frac{1}{c^{2}} \frac{\partial^{2} \phi}{\partial t^{2}}-\nabla^{2} \phi=0$

$m$ is free particle's mass

If we write wave function as solution of Klein-Gordon equation for free particle,[3]

$\phi=A_{0} \exp i(\omega t-\vec{k} \cdot \vec{x})$

$A_{0}$ is amplitude, $\omega$ is angular frequency, $k=|\vec{k}|$ is wave number

Energy and momentum is in inertial frame,[3]

$E=\hbar \omega, \vec{p}=\hbar \vec{k}$

Hence, energy-momentum relation is[3]

$E^{2}=\hbar^{2} \omega^{2}=p^{2} C^{2}+m^{2} C^{4}=\hbar^{2} k^{2} C^{2}+m^{2} C^{4}$

Or angular frequency- wave number relation is

$$
\frac{\omega^{2}}{c^{2}}=k^{2}+\frac{m^{2} c^{2}}{\hbar^{2}}
$$

2. Klein-Gordon Equation And Wave Function for Free Particle Field in RindlerSPACE-TIME

Rindler coordinates are

$$
\begin{aligned}
& c t=\left(\frac{c^{2}}{a_{0}}+\xi^{1}\right) \sinh \left(\frac{a_{0}}{c} \xi^{0}\right), x=\left(\frac{c^{2}}{a_{0}}+\xi^{1}\right) \cosh \left(\frac{a_{0}}{c} \xi^{0}\right)-\frac{c^{2}}{a_{0}} \\
& y=\xi^{2}, z=\xi^{3}
\end{aligned}
$$

We know Klein-Gordon equation in Rindler space-time. 
Klein-Gordon equation is for free particle field $\phi_{\xi}$ in Rindler space-time,[1]

$$
\frac{m^{2} c^{2}}{\hbar^{2}} \phi_{\xi}+\frac{1}{c^{2}} \frac{1}{\left(1+\frac{a_{0}}{c^{2}} \xi^{1}\right)^{2}} \frac{\partial^{2} \phi_{\xi}}{\left(\partial \xi^{0}\right)^{2}}-\nabla_{\xi}^{2} \phi_{\xi}-\frac{\partial \phi_{\xi}}{\partial \xi^{1}} \frac{a_{0}}{c^{2}} \frac{1}{\left(1+\frac{a_{0}}{c^{2}} \xi^{1}\right)}=0
$$

$m$ is free particle's mass

For we write wave function as solution of Klein-Gordon equation for free particle in Rindler spacetime, if we insert $\operatorname{Eq}(6)$ in $\operatorname{Eq}(2),[2,3]$

$$
\begin{aligned}
& \phi=A_{0} \exp i(\omega t-\vec{k} \cdot \vec{x}) \\
& =\phi_{\xi}=A_{0} \exp i\left[\left(\frac{c^{2}}{a_{0}}+\xi^{1}\right)\left\{\frac{\omega}{c} \sinh \left(\frac{a_{0} \xi^{0}}{c}\right)-k_{1} \cosh \left(\frac{a_{0} \xi^{0}}{c}\right)\right\}+k_{1} \frac{c^{2}}{a_{0}}-k_{2} \xi^{2}-k_{3} \xi^{3}\right]
\end{aligned}
$$

$\mathrm{Eq}(8)$ is the solution's function of the wave equation, $\mathrm{Eq}(7)$ in Rindler space-time.

In this point, energy-momentum transformation is[2]

$$
\begin{aligned}
& E=\hbar \omega=E_{\xi} \cosh \left(\frac{a_{0} \xi^{0}}{c}\right)+p_{\xi^{1}} C \sinh \left(\frac{a_{0} \xi^{0}}{c}\right) \\
& =\hbar \omega_{\xi} \cosh \left(\frac{a_{0} \xi^{0}}{c}\right)+\hbar k_{\xi^{1}} C \sinh \left(\frac{a_{0} \xi^{0}}{c}\right) \\
& p_{x}=\hbar k_{1}=\frac{E_{\xi}}{c} \sinh \left(\frac{a_{0} \xi^{0}}{c}\right)+p_{\xi^{1}} \cosh \left(\frac{a_{0} \xi^{0}}{c}\right)=\hbar \frac{\omega_{\xi}}{c} \sinh \left(\frac{a_{0} \xi^{0}}{c}\right)+\hbar k_{\xi^{1}} \cosh \left(\frac{a_{0} \xi^{0}}{c}\right) \\
& p_{y}=\hbar k_{2}=p_{\xi^{2}}=\hbar k_{\xi^{2}}, p_{z}=\hbar k_{3}=p_{\xi^{3}}=\hbar k_{\xi^{3}}
\end{aligned}
$$

In this time, we suppose $E_{\xi}=\hbar \omega_{\xi}, \vec{p}_{\xi}=\hbar \vec{k}_{\xi}$.In this careful point is we know $\omega, \vec{k}$ are constant.

But, in $\mathrm{Eq}(9), \mathrm{E}(10), \omega_{\xi^{\prime}}, k_{\xi^{1}}$ are variable functions with $\xi^{0}$. Hence, $\omega_{\xi}=\omega_{\xi}\left(\xi^{0}\right), k_{\xi^{1}}=k_{\xi^{1}}\left(\xi^{0}\right)$ don't have to use in $\operatorname{Eq}(7), \mathrm{Eq}(8)$. Energy-momentum relation is Rindler space-time,

$$
E_{\xi}^{2}-p_{\xi}^{2} C^{2}=\hbar^{2} \omega_{\xi}^{2}-\hbar^{2} k_{\xi}^{2} C^{2}=m^{2} C^{4}=E^{2}-p^{2} c^{2}=\hbar^{2} \omega^{2}-\hbar^{2} k^{2} C^{2}
$$

\section{CONCLUSION}

We found the wave function of Klein-Gordon's free particle in Rindler space-time. The wave function cannot use as a probability amplitude. In this paper, the particle has to do a spinless particle.

\section{REFERENCES}

[1]S.Yi, "Vibration of Yukawa Dependent Time and Extended Klein-Gordon Equation in Rindler Space-Time", International Journal of Advanced Research in Physical Science,7,7(2020)

[2]S.Yi, "Electromegnetic Wave Function and Equation, Lorentz Force in Rindler Space-time", International Journal of Advanced Research in Physical Science,5,9(2018)

[3]Klein-Gordon equation-Wikipedia 
[4]J.M. Normand, A Lie group: Rotations in Quantum Mechanics( North-Holland Pub. Co., 1980)

[5]J.D. Bjorken \& S. D. Drell, Relativistic Quantum Field(McGraw- Hill Co., 1965)

[6]P.Bergman,Introduction to the Theory of Relativity(Dover Pub. Co.,Inc., New York, 1976),Chapter V

[7]R.L.Liboff, Quantum Mechanics(Addison-Wesley Publishing Co., Inc.,1990)

[8]A.Beiser, Concept of Modern Physics(McGraw-Hill,Inc.,1991)

Citation: Sangwha-Yi, (2020). Klein-Gordon Equation and Wave Function for Free Particle in Rindler Space-Time. International Journal of Advanced Research in Physical Science (IJARPS) 7(9), pp. 10-12 2020.

Copyright: (C) 2020, Authors, This is an open-access article distributed under the terms of the Creative Commons Attribution License, which permits unrestricted use, distribution, and reproduction in any medium, provided the original author and source are credited. 